\title{
ALMA hints at the existence of an unseen reservoir of diffuse molecular gas in the Galactic bulge
}

\author{
M. Gerin ${ }^{1}$ and H. Liszt ${ }^{2}$ \\ ${ }^{1}$ LERMA, Observatoire de Paris, PSL Research University, CNRS, Sorbonne Universités, UPMC Univ. Paris 06, \\ École Normale Supérieure, 75005 Paris, France \\ e-mail: maryvonne.gerin@ens. fr \\ 2 National Radio Astronomy Observatory, 520 Edgemont Road, Charlottesville, VA 22903, USA \\ e-mail: hliszt@nrao.edu
}

Received 5 January 2017 / Accepted 6 February 2017

\begin{abstract}
Aims. We aim to understand the unexpected presence of mm-wave molecular absorption at $-200 \mathrm{~km} \mathrm{~s}^{-1}<v<-140 \mathrm{~km} \mathrm{~s}^{-1}$ in a direction that is well away from regions of the Galactic bulge where $\mathrm{CO}$ emission at such velocities is prominent.

Methods. We compared $89 \mathrm{GHz}$ Cycle 2 ALMA absorption spectra of $\mathrm{HCO}^{+}, \mathrm{HCN}$, and $\mathrm{HNC}$ toward the extragalactic continuum source B1741-312 at $l=-2.14^{\circ}, b=-1.00^{\circ}$ with existing CO, H I, and dust emission and absorption measurements. We placed the atomic and molecular gas in the bulge and disk using circular and non-circular galactic kinematics, deriving $N(\mathrm{H} \mathrm{I})$ from a combination of $21 \mathrm{~cm}$ emission and absorption and we derive $N\left(\mathrm{H}_{2}\right)$ from scaling of the $\mathrm{HCO}^{+}$absorption. We then inverted the variation of near-IR reddening $E(J-K)$ with distance modulus and scale $E(J-K)$ to a total gas column density $N(\mathrm{H})$ that may be compared to $N(\mathrm{H} \mathrm{I})$ and $N\left(\mathrm{H}_{2}\right)$.

Results. At galactocentric radii $R_{\text {gal }}>1.5 \mathrm{kpc}$, conventional measures such as the standard $\mathrm{CO}-\mathrm{H}_{2}$ conversion factor and locally observed $N\left(\mathrm{HCO}^{+}\right) / N\left(\mathrm{H}_{2}\right)$ ratio separately imply that $\mathrm{H} \mathrm{I}$ and $\mathrm{H}_{2}$ contribute about equally to $N(\mathrm{H})$, and the gas-derived $N(\mathrm{H})$ values are in broad agreement with those derived from $E(J-K)$. Within the Galactic bulge at $R_{\text {gal }}<1.5 \mathrm{kpc}, \mathrm{H} \mathrm{I}$ contributes less than $10 \%$ of the material inferred from $E(J-K)$, so that the molecular absorption detected here is needed to understand the extinction.
\end{abstract}

Key words. Galaxy: bulge - ISM: atoms - ISM: molecules

\section{Introduction}

The distribution of molecular gas in the Galactic nucleus has been heavily studied in the so-called central molecular zone (CMZ; Morris \& Serabyn 1996) that extends $\pm 1.2^{\circ}$ in Galactic longitude or $\pm 180 \mathrm{pc}$ projected distance at the IAU standard distance $R_{0}=8.5 \mathrm{kpc}$. The molecular component outside the CMZ, but within the bulge, is far less well understood owing to limited latitude coverage and sparse sampling of the $\mathrm{CO}$ survey (Bitran et al. 1997) and to uncertainty in the conversion from CO brightness to $\mathrm{H}_{2}$ column density (Bolatto et al. 2013).

In the Galactic bulge, the atomic gas has been fully if somewhat coarsely mapped (Burton \& Liszt 1983; HI4PI Collaboration et al. 2016), and the mass of $\mathrm{H} \mathrm{I}, 3 \times 10^{7} M_{\odot}$ is comparable to that of the molecular gas within the much more compact CMZ. The atomic gas shows a broad range of phenomena associated with large-scale gas flow in the Galactic bar outside the CMZ (Fux 1999) but only a small portion of that is seen in $\mathrm{CO}$ and other molecules (Rodriguez-Fernandez et al. 2006). Molecular emission is present in a few very broad-lined features associated with the shredding and intake of molecular gas at the standing dust lane shock in the Galactic bar, and CO is detected in the terminal-velocity feature associated with inward gas flow along the dust lane (Liszt 2006, 2008). Even CO emission is largely absent from most of the velocity range observed in $\mathrm{H} \mathrm{I}$, however, presumably because of limited sensitivity to weak, broad emission. This led to the suggestion (Liszt \& Burton 1978) that a large reservoir of molecular gas had yet to be observed in the bulge.
Some of this gas may have been serendipitously observed in absorption in an ALMA experiment studying the chemistry of $\mathrm{CF}^{+}$and $\mathrm{HOC}^{+}$in local diffuse molecular gas, and this paper explains why that might be the case. The structure of this work is as follows. In Sect. 2 we describe the new and existing observational material that is discussed. In Sect. 3 we present our observational results, from which we derive column densities of diffuse atomic and molecular gas in the Galactic disk and bulge, and compare these with gas column densities implied by the variation of near-IR dust reddening $E(J-K)$ with distance modulus. Section 4 is a summary and discussion.

\section{Observations and data reduction}

\subsection{ALMA absorption measurements}

We observed $\mathrm{HCO}^{+}, \mathrm{HCN}$, and $\mathrm{HNC}$ in absorption near $89 \mathrm{GHz}$ in ALMA Cycle 2 project 2013.1.01194.S, whose scientific goal was a comparative study of the chemistries of $\mathrm{CF}^{+}$and $\mathrm{HOC}^{+}$ with respect to $\mathrm{HF}$ and $\mathrm{H}_{2} \mathrm{O}$ in diffuse molecular gas. The blazar background source B1741-312 at $l=-2.1366^{\circ}, b=-0.9968^{\circ}$ was observed in nine spectral line windows, three of which covered the $J=1-0$ transitions of $\mathrm{HCN}, \mathrm{HCO}^{+}$, and $\mathrm{HNC}$ at $88.631 \mathrm{GHz}, 89.188 \mathrm{GHz}$, and $90.663 \mathrm{GHz}$, respectively, as discussed here. The appearance of the HCN absorption is complicated by the inherent hyperfine splitting into three components with local thermal equilibrium (LTE) optical depths in the ratio 1:5:3 in velocity order. Observations of $\mathrm{HOC}^{+}, \mathrm{CF}^{+}, \mathrm{C}_{3} \mathrm{H}^{+}$, 
and $\mathrm{c}-\mathrm{C}_{3} \mathrm{H}$ in the nearby gas will be discussed in a forthcoming paper.

Although the intended subject of study toward B1741-312 was relatively local gas near 0-velocity, the spectral setup was held in common with those used for Sgr A and Sgr B in the same science goal, therefore extending almost to $-200 \mathrm{~km} \mathrm{~s}^{-1}$ : the lowest-frequency transition, that of $\mathrm{HCN}$, extends the farthest to negative velocity, detecting (with $\mathrm{HCO}^{+}$) a feature at $-190 \mathrm{~km} \mathrm{~s}^{-1}$ that was narrowly missed in the highest frequency spectral window, that for HNC. The channel spacing of the ALMA data presented here is $244 \mathrm{kHz}$, or approximately $0.8 \mathrm{~km} \mathrm{~s}^{-1}$, and the resolution is twice as high.

The continuum flux was $0.59 \mathrm{Jy}$ and the source was observed for 720 s, leading to channel-channel rms optical depth fluctuations of 0.0062 at zero optical depth. The spectra were reduced using the standard ALMA pipeline in January and February 2016 after the data had been delivered. Spectra were extracted from the final data cubes at the pixel of peak continuum flux.

The velocities of these and all other data discussed here are measured with respect to the kinematic definition of the Local Standard of Rest (LSR).

\subsection{Other data: $\mathrm{HI}$ and $\mathrm{CO}$ emission}

We present CO $J=1-0$ emission profiles from the Southern Mini-Telescope CO survey of Bitran et al. (1997) at $8^{\prime}$ spatial resolution on a $1 / 8^{\circ}$ grid and $\mathrm{H}$ I emission from the Southern Galactic Plane Survey (SGPS) survey data of McClure-Griffiths et al. (2005) at 145" spatial resolution, which we used in conjunction with the $\mathrm{H}$ I absorption line profile of Dickey et al. (1983) to derive an optical-depth corrected $N(\mathrm{H} \mathrm{I})$ as

$N(\mathrm{H} \mathrm{I})=1.823 \times 10^{18} \mathrm{~cm}^{-2} \int \frac{\tau(v) T_{\mathrm{B}}(v)}{1-\exp (-\tau(v))} \mathrm{d} v$,

where $\tau$ and $T_{\mathrm{B}}$ are the optical depth and brightness temperature and the units of velocity are $\mathrm{km} \mathrm{s}^{-1}$.

\subsection{Extinction}

We also use data at $6^{\prime}$ resolution from the 3D IR extinction map of the bulge of Schultheis et al. (2014), extracting the variation of $E(J-K)$ along the line of sight at $(l, b)=\left(-2.1^{\circ},-1.0^{\circ}\right)$ and using the relationships $A_{\mathrm{K}} / A_{\mathrm{V}}=0.11, A_{\mathrm{K}} / E(J-K)=0.689$, or $A_{\mathrm{V}}=6.26 E(J-K)$ and $N(\mathrm{H})=1.171 \times 10^{22} \mathrm{~cm}^{-2} E(J-K)$, where $N(\mathrm{H})$ is the total column density of $\mathrm{H}$-nuclei in atomic and molecular form, $N(\mathrm{H})=N(\mathrm{H} \mathrm{I})+2 \mathrm{~N}\left(\mathrm{H}_{2}\right)$.

\section{Observational results}

Atomic and molecular absorption and emission spectra toward and around B1741-312 are shown in Fig. 1. Strong molecular absorption lines are seen from local gas around 0-velocity, and the molecular feature at $v=7 \mathrm{~km} \mathrm{~s}^{-1}$ corresponds to a strong $\mathrm{H}$ I selfabsorption feature originally discovered by Heeschen (1955) that provided the first evidence of inhomogeneity in the temperature structure of the interstellar H I gas. The high column density of local gas seen around 0-velocity dominates the total absorption and emission in this direction, but molecular absorption also appears around $-25,-150$, and $-190 \mathrm{~km} \mathrm{~s}^{-1}$. The spectrum of $\mathrm{HNC}$, lying at the highest rest frequency, did not quite extend out to the narrow absorption near $-190 \mathrm{~km} \mathrm{~s}^{-1}$.

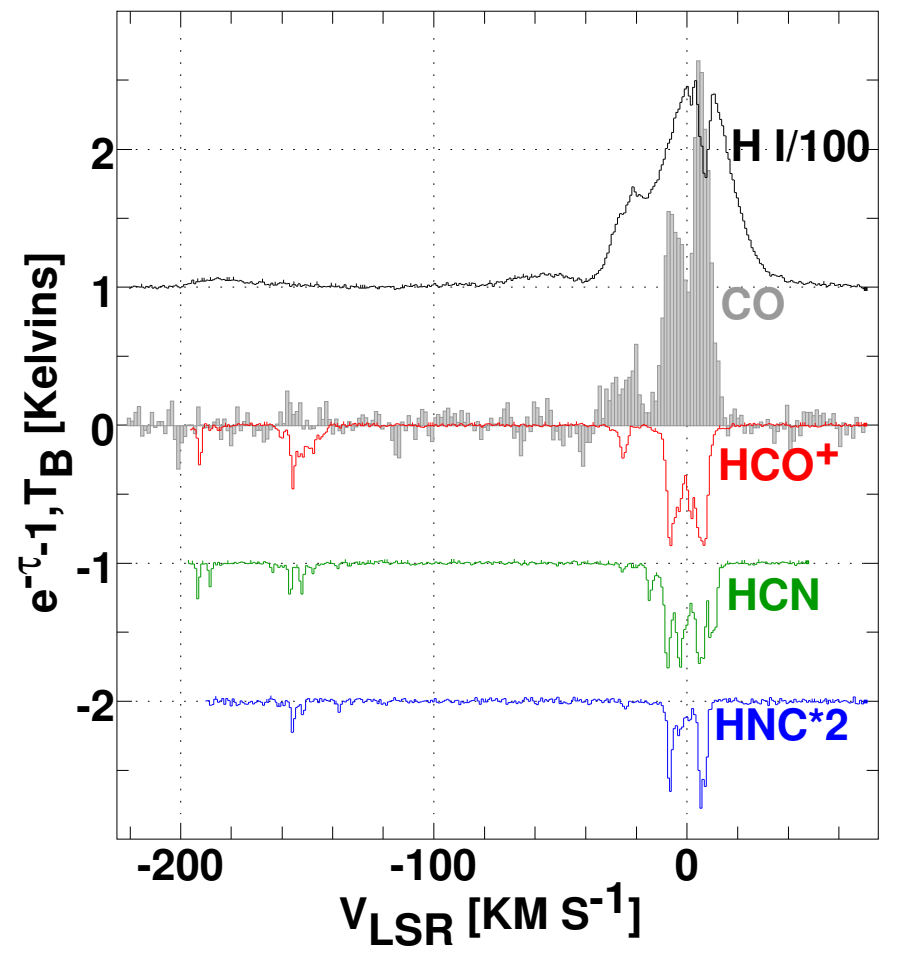

Fig. 1. SGPS H I, Southern Mini CO, and ALMA $\mathrm{HCO}^{+}, \mathrm{HCN}$, and $\mathrm{HNC}$ profiles. The strongest $\mathrm{CO}$ emission feature is the counterpart of a strong and very widespread $\mathrm{H}$ I self-absorption feature.

Molecular absorption at $-190 \mathrm{~km} \mathrm{~s}^{-1}$ from the Galactic bulge is narrow in $\mathrm{HCO}^{+}$and $\mathrm{HCN}$, as perhaps arising from a single isolated cloud. The absorption around $-150 \mathrm{~km} \mathrm{~s}^{-1}$ is wider and more complex, with two kinematic sub-components separated by $6 \mathrm{~km} \mathrm{~s}^{-1}$ in HNC and more continuous absorption over $20 \mathrm{~km} \mathrm{~s}^{-1}$ in $\mathrm{HCO}^{+}$. The absorption depth ratios of the three species and the somewhat greater ubiquity of $\mathrm{HCO}^{+}$in the high negativevelocity bulge features are typical of molecular absorption from diffuse molecular gas in the interstellar medium (ISM) near the Sun (Liszt \& Lucas 2001), or the Galactic disk (Godard et al. 2010).

$\mathrm{H}$ I emission is apparent around $-190 \mathrm{~km} \mathrm{~s}^{-1}$ toward B1741312 in Fig. 1, but is seen only much more weakly at $-150 \mathrm{~km} \mathrm{~s}^{-1}$. $\mathrm{CO}$ emission is detected at $-22 \mathrm{~km} \mathrm{~s}^{-1}$ and with low significance at $-150 \mathrm{~km} \mathrm{~s}^{-1}$, but not at $-190 \mathrm{~km} \mathrm{~s}^{-1}$. CO emission at high negative velocities is, however, present in the vicinity of B1741312. In Fig. 2 we superpose the $\mathrm{HCO}^{+}$absorption profile on a latitude-velocity cut at $l=-2.125^{\circ}$ in $\mathrm{CO}$ emission, illustrating the peculiar geometry and non-circular kinematics of gas in the bulge. $\mathrm{CO}$ emission from the bulge, at $|v|>30 \mathrm{~km} \mathrm{~s}^{-1}$, appears preferentially above the Galactic equator owing to the tip and tilt of the inner galaxy gas (Burton \& Liszt 1978; Liszt \& Burton 1978) with the high positive-velocity gas on the far side of the gas distribution appearing at higher latitude ${ }^{1}$. CO emission around $-190 \mathrm{~km} \mathrm{~s}^{-1}$ is present in Fig. 2 only at $b>-0.5^{\circ}$ at a minimum projected distance of $75 \mathrm{pc}$.

The distribution of $\mathrm{CO}$ emission is shown more comprehensively in Fig. 3, which locates B1741-312 on the sky as seen in slices $20 \mathrm{~km} \mathrm{~s}^{-1}$ wide of the $\mathrm{CO}$ emission data cube. CO emission is detected at $-190 \mathrm{~km} \mathrm{~s}^{-1}$ within some $10-15^{\prime}$ of the

$1 \mathrm{CO}$ emission at $-54 \mathrm{~km} \mathrm{~s}^{-1}$ that is centered on the Galactic equator arises in the so-called near $3 \mathrm{kpc}$ arm (Dame \& Thaddeus 2008) and does not share the tilted geometry. 


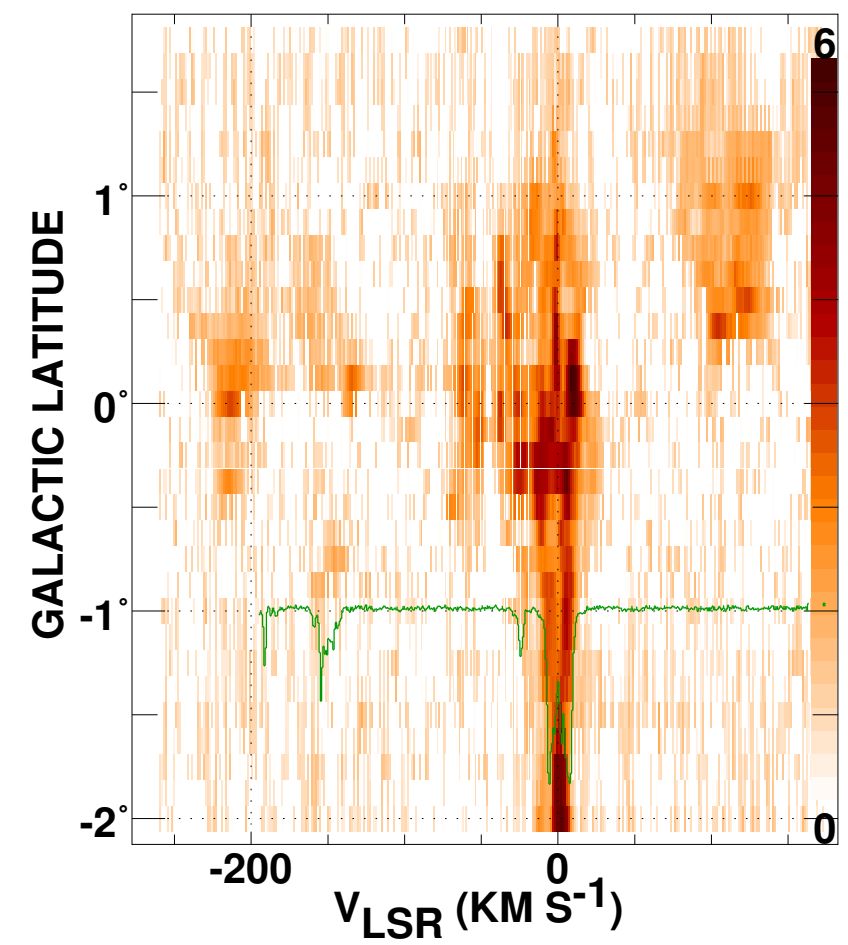

Fig. 2. Latitude-velocity diagram of $\mathrm{CO}$ emission at $l=-2.125^{\circ}$ with the ALMA $\mathrm{HCO}^{+}$absorption profile superposed such that its 0-level is at the latitude of B1741-312.

position of B1741-312, a projected separation of 25-35 pc. The molecular absorption at $-150 \mathrm{~km} \mathrm{~s}^{-1}$ is seen just at the periphery of a nearby $\mathrm{CO}$ emission feature, as also suggested in Fig. 2.

\subsection{Placement of kinematic features}

Gas kinematics in the Galactic disk are dominated by circular rotation while motions in the Galactic bar and bulge are characterized by strongly non-circular velocities (Fux 1999). Figure 4 shows the variation in LSR velocity with distance and galactocentric radius for pure circular motion with a flat rotation curve with $R_{0}=8.5 \mathrm{kpc}, \Theta=220 \mathrm{~km} \mathrm{~s}^{-1}$, and for a non-circular velocity field inside $R_{\text {gal }}=1.5 \mathrm{kpc}$ using approximately equal circular and radial motions $170-180 \mathrm{~km} \mathrm{~s}^{-1}$ to explain the geometry and gas motions in the inner galaxy, including the stronger CO emission at higher latitude in Fig. 2 (Burton \& Liszt 1978). In pure rotation, velocities $v<-140 \mathrm{~km} \mathrm{~s}^{-1}$ arise exclusively within $300-500 \mathrm{pc}$ of the center: with non-circular motion the velocity field is bifurcated, and velocities $v<-140 \mathrm{~km} \mathrm{~s}^{-1}$ arise at both larger and smaller radii in the region interior to $R_{\mathrm{gal}}=$ $1.5 \mathrm{kpc}$ (the outermost radius of the underlying model).

The line-of-sight velocity gradient for disk gas in pure rotation is quite shallow within $5-6 \mathrm{kpc}$ of the Sun, and the LSR velocity at the edge of the bulge gas distribution at $R_{\text {gal }}=1.5 \mathrm{kpc}$ in Fig. 4 is $-40 \mathrm{~km} \mathrm{~s}^{-1}$. A separation into bulge gas at $v \lesssim$ $-140 \mathrm{~km} \mathrm{~s}^{-1}$ and disk gas at $v \gtrsim-40 \mathrm{~km} \mathrm{~s}^{-1}$ works quite well for the molecules seen toward B1741-312 and for most of the $\mathrm{H}$ I because there are no features or strong emission in the intermediate velocity range. $\mathrm{H} \mathrm{I}$ emission from the $3 \mathrm{kpc}$ arm is seen in the intermediate velocity range, and the $3 \mathrm{kpc}$ arm is usually placed in the transition region between the bulge and disk gas distributions (Fux 1999).

\subsection{Derived results}

To provide a quantitative assessment of the importance of the molecular absorption, Table 1 summarizes the observed line properties for a set of velocity ranges framing the features that were detected in $\mathrm{HCO}^{+}$. The table entries from the top down are as follows:

- $R_{\text {gal }}$ : galactocentric radius. For the two most negative velocity ranges, the gas is ascribed to the bulge at $R_{\mathrm{gal}}=$ $320-1500$ pc without greater specificity given the bifurcation in the velocity shown in Fig. 4 and discussed in Sect. 3.1: $320 \mathrm{pc}$ is the pericenter distance. The other ranges of galactocentric radius were assigned on the basis of the flat rotation curve with $R_{0}=8.5 \mathrm{kpc}, \Theta=220 \mathrm{~km} \mathrm{~s}^{-1}$. The identical radius ranges given for the two highest velocity intervals both correspond to $v \geq-15 \mathrm{~km} \mathrm{~s}^{-1}$ in pure rotation. The molecular gas seen at $7 \mathrm{~km} \mathrm{~s}^{-1}$ must be near the Sun, given the strong $\mathrm{H}$ I self-absorption, even though positive velocities are not allowed by rotational kinematics within the solar circle toward B1741-312. H I emission at $v \gtrsim 20 \mathrm{~km} \mathrm{~s}^{-1}$ probably arises in the inner galaxy at an uncertain galactocentric radius, and its contribution to the column density, $7.3 \%$ of the total, has been ignored in this table.

- $W_{\mathrm{X}}$ : the integrated observed optical depth for $\mathrm{HCN}, \mathrm{HCO}^{+}$, and $\mathrm{HNC}$ (units of $\mathrm{km} \mathrm{s}^{-1}$ ) and integrated brightness temperature for $\mathrm{H} \mathrm{I}$ and $\mathrm{CO}$ observed in emission, in units of $\mathrm{K}-\mathrm{km} \mathrm{s}^{-1}$.

- $N(\mathrm{X})$ : column densities (units of $\mathrm{cm}^{-2}$ ) calculated using Eq. (1) for $\mathrm{H} \mathrm{I}, T_{\mathrm{ex}}=2.729 \mathrm{~K}$ for $\mathrm{HCO}^{+}$, and with $N\left(\mathrm{HCO}^{+}\right) / N\left(\mathrm{H}_{2}\right)=3 \times 10^{-9}$, the local value for solar metallicity as a benchmark for $\mathrm{H}_{2}$. A fixed conversion taken from the values determined near the Sun was also used by Schultheis et al. (2014), whose 3D $E(J-K)$ extinction maps we used to derive the variation of $N(\mathrm{H})$ as inferred from dust measures.

- $X_{\mathrm{CO}}: N\left(\mathrm{H}_{2}\right) / W_{\mathrm{CO}}$, the $\mathrm{CO}-\mathrm{H}_{2}$ conversion factor. We note that the assumed $\mathrm{HCO}^{+}$abundance yields a canonical value $2 \times$ $10^{20} \mathrm{H}_{2} \mathrm{~cm}^{-2}\left(\mathrm{~K}-\mathrm{km} \mathrm{s}^{-1}\right)^{-1}$ for the $\mathrm{CO}-\mathrm{H}_{2}$ conversion factor for the two velocity intervals at $v \geq-15 \mathrm{~km} \mathrm{~s}^{-1}$, independent of any assumption about the rotation curve or placement of the gas in the Galaxy. Schultheis et al. (2014) derived a similar $\mathrm{CO}-\mathrm{H}_{2}$ conversion factor in the vicinity of B1741312 when $N(\mathrm{H})$ was derived from the total $E(J-K)$ and compared with profile-integrated total $\mathrm{CO}$ measurements from the NANTEN2 telescope. This occurs because the gas column is dominated by the local material seen around zero-velocity.

The tabulated values of $N(\mathrm{H} \mathrm{I})$ and $N\left(\mathrm{H}_{2}\right)$ show a marked change from an approximately equal mixture of atomic and molecular gas in the disk at $R_{\text {gal }}>3 \mathrm{kpc}$ to a predominantly molecular medium in the bulge at $R_{\text {gal }}<1.5 \mathrm{kpc}$. Deriving the $\mathrm{H}_{2}$ column density from $\mathrm{CO}$ emission using a standard $\mathrm{CO}-$ $\mathrm{H}_{2}$ conversion factor $N\left(\mathrm{H}_{2}\right) / W_{\mathrm{CO}}=2 \times 10^{20} \mathrm{~cm}^{-2}\left(\mathrm{~K}-\mathrm{km} \mathrm{s}^{-1}\right)^{-1}$ (Bolatto et al. 2013) also yields approximately equal proportions locally, but $\mathrm{CO}$ emission underrepresents the molecular gas at $R_{\text {gal }}<1.5 \mathrm{kpc}$. This is in strong contrast to the low values for the $\mathrm{CO}-\mathrm{H}_{2}$ conversion factor that apply in the $\mathrm{CMZ}$ where $\mathrm{CO}$ is overluminous (Bolatto et al. 2013; Schultheis et al. 2014). The transition region at $R_{\mathrm{gal}}=1.5-3 \mathrm{kpc}$ represented by the velocity range $-41 \mathrm{~km} \mathrm{~s}^{-1} \leq v \leq-15 \mathrm{~km} \mathrm{~s}^{-1}$ is predominantly atomic when $N\left(\mathrm{H}_{2}\right)$ is derived from $\mathrm{HCO}^{+}$, but with relatively bright $\mathrm{CO}$, leading to a small implied $\mathrm{CO}-\mathrm{H}_{2}$ conversion factor. The proportion of molecular gas would be much larger and about 


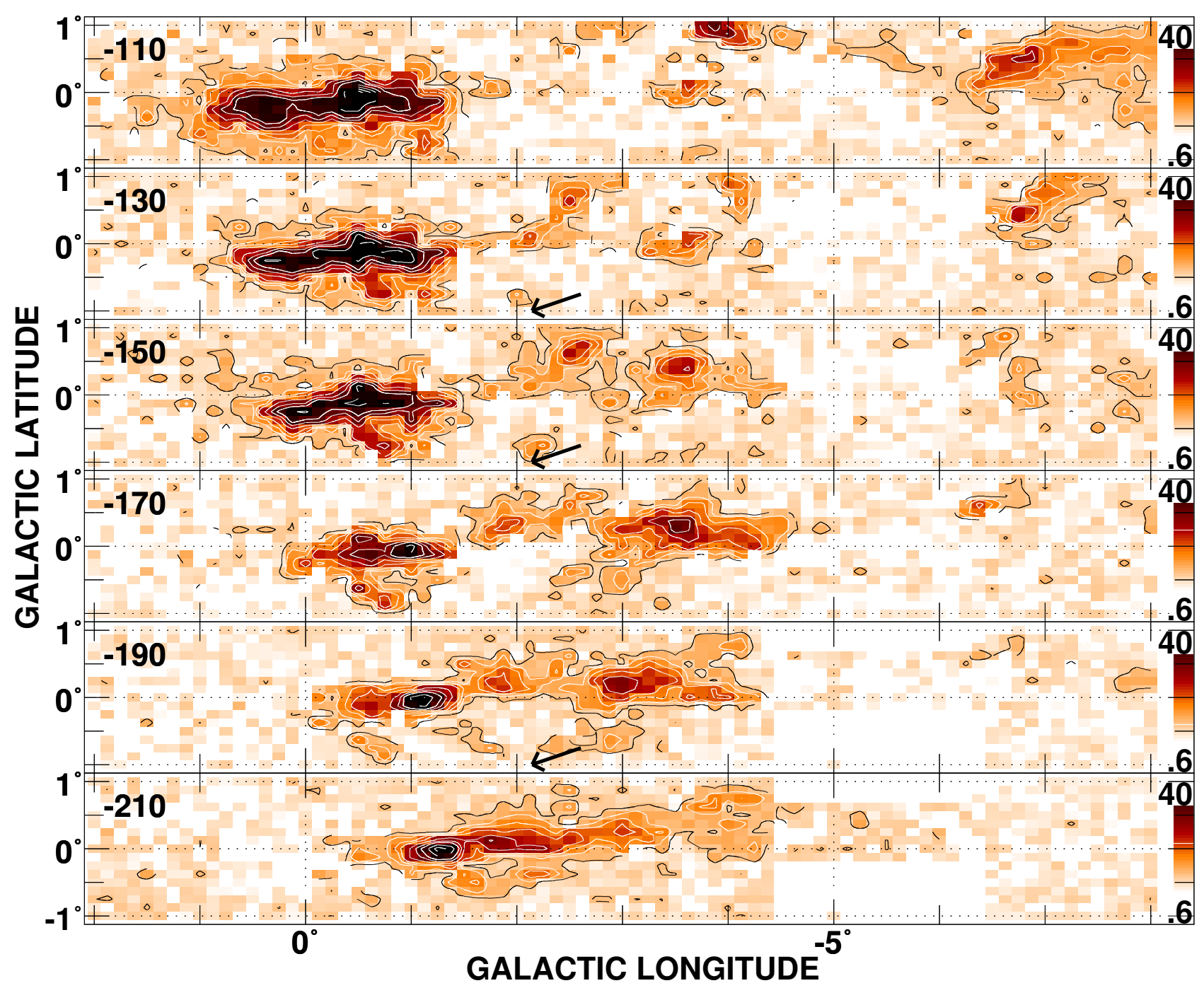

Fig. 3. CO integrated intensity maps in $20 \mathrm{~km} \mathrm{~s}^{-1}$ intervals centered as indicated in each panel. An arrow marks the position of B1741-312 at $l=-2.1366^{\circ}, b=-0.9968^{\circ}$ in panels where molecular absorption was detected. The color scale extends from 0.5 to $40 \mathrm{~K}-\mathrm{km} \mathrm{s}^{-1}$. Contours are shown at $2,4,7,11,20,30,40,60,80,100$, and $120 \mathrm{~K}-\mathrm{km} \mathrm{s}^{-1}$.

equal to that of $\mathrm{H} \mathrm{I}$ if the standard local $\mathrm{CO}-\mathrm{H}_{2}$ conversion were applied.

\subsection{Importance of the molecular absorption}

In Fig. 5 we show the variation of cumulative total column density $N(\mathrm{H})$ working outward to the solar circle, as derived under various assumptions. The solid curve results from interpolating between the binned entries in the $3 \mathrm{D}$ extinction data cube of Schultheis et al. (2014) at $l=-2 \cdot 1^{\circ}, b=-1^{\circ}$ using a constant scaling $N(\mathrm{H})=1.171 \times 10^{22} \mathrm{~cm}^{-2} E(J-K)$, as discussed in Sect. 2.3. The extinction data do not extend beyond a heliocentric distance of $10.5 \mathrm{kpc}$, but this should include the entire bulge contribution, while only a very small contribution is expected from the far-side disk gas at the latitude of B1741-312. Even the radio spectra do not sample much material outside the solar circle, which is seen at $z$-heights exceeding $300 \mathrm{pc}$ on the far side of the Galaxy.

We also show in Fig. 5 values for $N(\mathrm{H} \mathrm{I})$ and $N(\mathrm{H})$ where the latter is derived either using a constant abundance $X\left(\mathrm{HCO}^{+}\right)=$ $3 \times 10^{-9}$ or a canonical CO- $\mathrm{H}_{2}$ conversion factor $N\left(\mathrm{H}_{2}\right) / W_{\mathrm{CO}}=$ $2 \times 10^{20} \mathrm{H}_{2} \mathrm{~cm}^{-2}\left(\mathrm{~K}-\mathrm{km} \mathrm{s}^{-1}\right)^{-1}$. The value plotted is integrated over the full corresponding velocity range. We do not pretend to know where within the range the matter is distributed.
The least uncertain values are those for $N(\mathrm{H} \mathrm{I})$, so that if the radii are properly assigned, it is apparent that the extinction requires an additional contribution whose magnitude is like that which we have seen in molecular absorption. In the outer regions of the disk, the existence of the molecular gas can be inferred from $\mathrm{CO}$ emission using a standard $\mathrm{CO}-\mathrm{H}_{2}$ conversion factor, but this is not the case in the bulge at $R_{\text {gal }}<1.5 \mathrm{kpc}$.

\subsection{High $\mathrm{H}_{2}$-fractions in the bulge gas}

Features associated with the bulge have a remarkably high fraction of hydrogen in molecular form, $\sim 0.8$ and $\sim 0.95$ for the -190 and $-150 \mathrm{~km} \mathrm{~s}^{-1}$ velocity components, respectively. Using the analytical framework for the $\mathrm{H} \mathrm{I} / \mathrm{H}_{2}$ transition developed by Sternberg et al. (2014) and Bialy \& Sternberg (2016), we derived the parameter $\frac{I_{\mathrm{UV}}}{n_{100}}$ where $I_{\mathrm{UV}}$ is the intensity of the FUV radiation field in units of the Draine field and $n_{100}=\frac{n(\mathrm{H})}{100 \mathrm{~cm}^{-3}}$ is the gas density in units of $100 \mathrm{H}$-nuclei $\mathrm{cm}^{-3}$. We find $\frac{I_{\mathrm{UV}}}{n_{100}} \sim 0.5$ and $\sim 0.2$ for these two clouds, indicating that they are exposed to a low far-ultraviolet (FUV) radiation field. We note that the density must be modest, $n_{100} \leq 1$, since no significant $\mathrm{CO}$ emission is detected. 


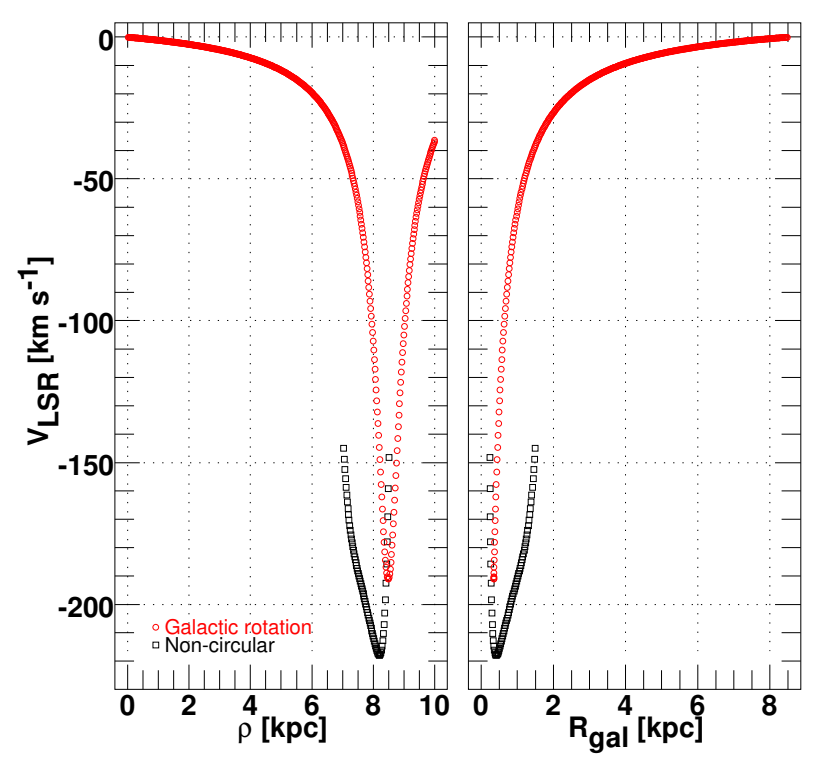

Fig. 4. Variation of LSR velocity with heliocentric distance (left) and galactocentric radius (right) for circular and non-circular velocity fields in the direction of B1741-312 at $l=-2.14^{\circ}, b=-1.0^{\circ}$. The curves labeled "Galactic rotation" are for a flat rotation curve with $R_{0}=8.5 \mathrm{kpc}$, $\Theta=220 \mathrm{~km} \mathrm{~s}^{-1}$, and the non-circular velocity field interior to $R_{\mathrm{gal}}=$ $1.5 \mathrm{kpc}$ is taken from Burton \& Liszt (1978). The diagrams were constructed by taking equal steps in heliocentric distance, and the density of points represents the line-of-sight geometry and velocity gradients.

The theory indicates that $\mathrm{H}_{2}$ is mostly protected by selfshielding in such an environment, with extinction contributed by dust grains providing a minor contribution to the protection of $\mathrm{H}_{2}$ against photodissociation. It is known from analysis of the spatial distribution of star-forming regions in the inner galaxy (e.g., Churchwell et al. 2009) that star formation is weak in the Galactic bulge, possibly as a consequence of the non-circular motions induced by the bar. The old stellar population of the bulge is expected to produce a radiation field dominated by visible and near-IR radiation, which can heat the dust (Robitaille et al. 2012) but is less effective at dissociating molecular hydrogen. The diffuse gas in the bulge therefore follows the general trend of matter in the disk, which has an increasing fraction of hydrogen in molecular form at smaller $R_{\text {gal }}$, more than $80 \%$ at $R_{\text {gal }} \approx 2 \mathrm{kpc}$ (Koda et al. 2016).

\subsection{Influence of metallicity variation}

Of course the tabulated values are affected by uncertainty in the metallicity in the bulge gas and disk gas, and an increased metallicity, if reflected in a higher $\mathrm{HCO}^{+}$relative abundance and dust/gas ratio, would lead to smaller $N\left(\mathrm{H}_{2}\right)$ inferred from $\mathrm{HCO}^{+}$ and $N(\mathrm{H})$ inferred from extinction. A factor three increase in $N\left(\mathrm{HCO}^{+}\right) / N\left(\mathrm{H}_{2}\right)$ would yield nearly equal atomic and molecular gas columns at $v=-190 \mathrm{~km} \mathrm{~s}^{-1}$, but no reasonable increase in metallicity would equalize the $\mathrm{H}_{2} / \mathrm{H}$ I ratio at $v=-150 \mathrm{~km} \mathrm{~s}^{-1}$, which is 37. We note that Schultheis et al. (2014), from whose work we drew the variation of $E(J-K)$, also used a constant gas-reddening ratio to derive $N(\mathrm{H})$ and $W_{\mathrm{CO}}$.

\subsection{Another estimate of the total atomic gas deficit}

The more recent value of the IRAS dust-inferred optical reddening $E_{B-V}$ in the direction of B1741 at $6^{\prime}$ resolution is

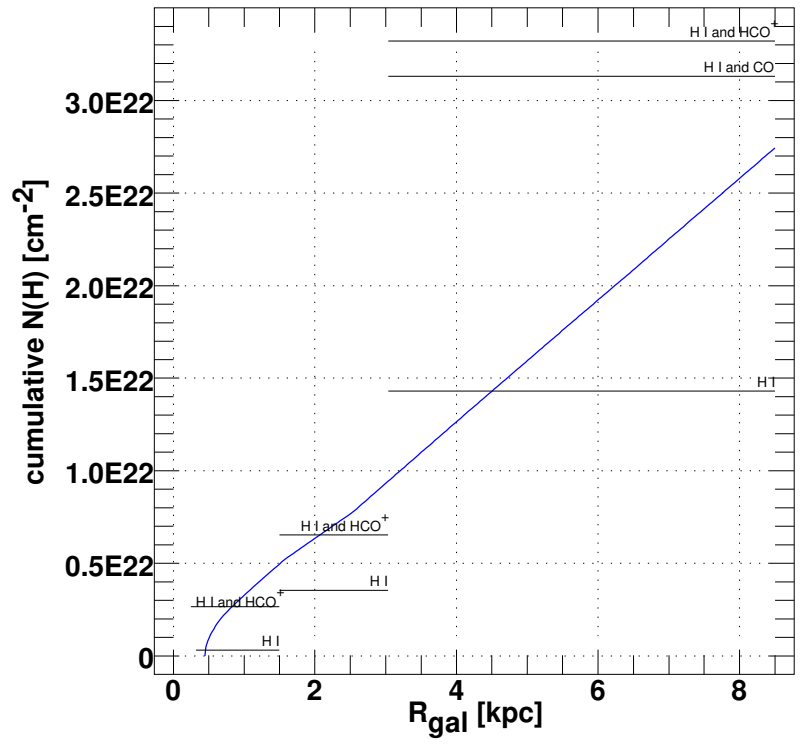

Fig. 5. Variation in cumulative hydrogen column densities $N(\mathrm{H} \mathrm{I})$ and $N(\mathrm{H})=N(\mathrm{H} \mathrm{I})+2 N\left(\mathrm{H}_{2}\right)$ vs. galactocentric radius. The solid blue curve for the total column density $N(\mathrm{H})$ was derived by inverting the variation of $E(J-K)$ with distance modulus in the 3D extinction data cube of Schultheis et al. (2014), as discussed in Sect. 2.3. The binned estimates for $N(\mathrm{H})$ from $\mathrm{H} \mathrm{I}$ and $\mathrm{HCO}^{+}$or $\mathrm{H} \mathrm{I}$ and $\mathrm{CO}$ assume $N\left(\mathrm{HCO}^{+}\right) / N\left(\mathrm{H}_{2}\right)=$ $3 \times 10^{-9}$ or $N\left(\mathrm{H}_{2}\right)=2 \times 10^{20} \mathrm{~cm}^{-2} W_{\mathrm{CO}}$. The bins correspond to the ranges of galactocentric radius listed in Table 1 .

$E_{B-V}=6.3$ mag (Schlafly \& Finkbeiner 2011), which is somewhat beyond the stated range of validity of this estimator. Converting this into total column density with a recent value of the $N(\mathrm{H}) / E_{B-V}$ ratio (Liszt $2014 \mathrm{~b}$, a; Hensley \& Draine 2017) yields a high total column density $N(\mathrm{H})=6.3 \times 8 \times 10^{21}=5.0 \times$ $10^{22} \mathrm{H}$-nuclei $\mathrm{cm}^{-2}$. The implied gas deficit $N(\mathrm{H})-N(\mathrm{H} \mathrm{I}) \approx$ $3.5 \times 10^{22} \mathrm{H}$-nuclei $\mathrm{cm}^{-2}$ is much higher than can be explained by either the $\mathrm{HCO}^{+}$absorption or the $\mathrm{CO}$ emission.

\section{Summary}

We began by noting the accidental observation of $\mathrm{mm}$ wave $\mathrm{HCO}^{+}, \mathrm{HCN}$ and $\mathrm{HNC}$ absorption at $-150 \mathrm{~km} \mathrm{~s}^{-1}$ and $-190 \mathrm{~km} \mathrm{~s}^{-1}$ toward the extragalactic mm-wave continuum background source B1741-312 seen at $l=-2.1366^{\circ}, b=$ $-0.9968^{\circ}$ (Fig. 1). There is weak $\mathrm{CO}$ emission in the vicinity of the position of B1741 (Figs. 2 and 3), but prominent CO emission at such velocities is confined to smaller longitudes and latitudes nearer the Galactic equator.

Emission and absorption at velocities $v \lessgtr-150 \mathrm{~km} \mathrm{~s}^{-1}$ cannot be precisely located along the line of sight owing to imprecise knowledge of the bar-like inner-galaxy velocity field, but must arise in gas located within the Galactic bulge (Fig. 4). Similarly, strong disk absorption and emission at $-40 \mathrm{~km} \mathrm{~s}^{-1} \leq v \leq$ $0 \mathrm{~km} \mathrm{~s}^{-1}$ can be understood in terms of a flat disk rotation velocity curve with $R_{0}=8.5 \mathrm{kpc}, \Theta=220 \mathrm{~km} \mathrm{~s}^{-1}$, but the bright emitting gas at $0 \mathrm{~km} \mathrm{~s}^{-1} \leq v \leq 20 \mathrm{~km} \mathrm{~s}^{-1}$ is not permitted by rotational kinematics and cannot be precisely located either, although we know that it must be nearby because it is self-absorbed in $\mathrm{H} \mathrm{I}$.

The atomic and molecular absorption profiles were interpreted on this basis in Table 1, where we assigned broad ranges in galactocentric radius to the kinematic intervals containing the observed molecular features. We derived column densities $N(\mathrm{H} \mathrm{I}), N\left(\mathrm{HCO}^{+}\right)$, and $N\left(\mathrm{H}_{2}\right)$ assuming as a baseline the locally-determined value $N\left(\mathrm{HCO}^{+}\right) / N\left(\mathrm{H}_{2}\right)=3 \times 10^{-9}$. 
Table 1. Observed and derived quantities.

\begin{tabular}{lccccc}
\hline \hline & & & Velocity range $\left(\mathrm{km} \mathrm{s}^{-1}\right)$ & $-15,-1$ & $-1,19$ \\
\hline Quantity & $-196,-180$ & $-164,-135$ & $-41,-15$ & $3035-8500$ & $3035-8500$ \\
\hline$R_{\text {gal }}[\mathrm{pc}]$ & $320-1500$ & $320-1500$ & $1500-3035$ & $1524(7.6)$ & $2622(10.4)$ \\
\hline$W_{\mathrm{HI}}\left[\mathrm{K} \mathrm{km} \mathrm{s}^{-1}\right]$ & $79.9(3.1)$ & $32.8(3.8)$ & $1062(6.1)$ & $9.10(0.03)$ & $12.22(0.32)$ \\
$W_{\mathrm{HCO}^{+}}\left[\mathrm{km} \mathrm{s}^{-1}\right]$ & $0.802(0.025)$ & $3.381(0.036)$ & $1.108(0.031)$ & $8.62(0.03)$ & $9.65(0.03)$ \\
$W_{\mathrm{HCN}}\left[\mathrm{km} \mathrm{s}^{-1}\right]$ & $0.640(0.027)$ & $1.310(0.036)$ & $0.444(0.032)$ & $1.42(0.03)$ & $1.72(0.03)$ \\
$W_{\mathrm{HNC}}\left[\mathrm{km} \mathrm{s}^{-1}\right]$ & & $0.333(0.034)$ & $0.062(0.031)$ & $14.53(0.42)$ & $22.37(0.49)$ \\
$W_{\mathrm{CO}}\left[\mathrm{K} \mathrm{km} \mathrm{s}^{-1}\right]$ & $0(0.44)$ & $1.40(0.69)$ & $5.55(0.50)$ & $4.05(0.02) \times 10^{21}$ & $6.16(0.02) \times 10^{21}$ \\
\hline$N(\mathrm{H} \mathrm{I})\left[\mathrm{cm}^{-2}\right]$ & $1.51(0.06) \times 10^{20}$ & $6.13(0.71) \times 10^{19}$ & $2.77(0.02) \times 10^{21}$ & $1.031(0.03) \times 10^{13}$ \\
$N\left(\mathrm{HCO}^{+}\right)\left[\mathrm{cm}^{-2}\right]$ & $8.58(0.26) \times 10^{11}$ & $3.62(0.03) \times 10^{12}$ & $1.18(0.03) \times 10^{12}$ & $9.74(0.28) \times 10^{12}$ & $1.31\left(10 \times 10^{21}\right.$ \\
$N\left(\mathrm{H}_{2}\right)\left[\mathrm{cm}^{-2}\right]$ & $2.86 \times 10^{20}$ & $1.21 \times 10^{21}$ & $3.92 \times 10^{20}$ & $3.25 \times 10^{21}$ & $4.36 \times 10^{21}$ \\
\hline$X_{\mathrm{CO}}\left[\mathrm{cm}^{-2} /{\left.\mathrm{K}-\mathrm{km} \mathrm{s}^{-1}\right]}^{>2.17 \times 10^{20, a}}\right.$ & $8.64 \times 10^{20}$ & $7.06 \times 10^{19}$ & $2.24 \times 10^{20}$ & $1.95 \times 10^{20}$ \\
\hline
\end{tabular}

Notes. See Sect. 2.3 for a description of table entries. ${ }^{(a)}$ Using the $3 \sigma$ upper limit for $W_{\mathrm{CO}}$.

This $\mathrm{HCO}^{+}$-based estimate of $N\left(\mathrm{H}_{2}\right)$ yields canonical values of the $\mathrm{CO}-\mathrm{H}_{2}$ conversion factor $N\left(\mathrm{H}_{2}\right) / W_{\mathrm{CO}}=2.0-2.2 \times$ $10^{20} \mathrm{H}_{2} \mathrm{~cm}^{-2}\left(\mathrm{~K}-\mathrm{km} \mathrm{s}^{-1}\right)^{-1}$ for either of the velocity intervals around $0 \mathrm{~km} \mathrm{~s}^{-1}$ in Table 4 .

Broadly speaking, the balance between the atomic and molecular gas constituents shifts from approximate equality in the disk to a mostly molecular medium within the bulge. Figure 5 shows the galactocentric radial distribution of total column density $N(\mathrm{H})$ derived by inverting the run of IR reddening $E(J-K)$ with distance modulus in the 3D extinction data cube of Schultheis et al. (2014) using a fixed relationship $N(\mathrm{H})=$ $1.171 \times 10^{22} \mathrm{~cm}^{-2} E(J-K)$ as described in Sect. 2.3. H I can account for only a small portion of the required gas column inside the bulge at $R_{\mathrm{gal}}<1.5 \mathrm{kpc}$. The gas shortfall can be explained by the observed $\mathrm{HCO}^{+}$absorption using the locally determined fractional abundance $N\left(\mathrm{HCO}^{+}\right) / N\left(\mathrm{H}_{2}\right)=3 \times 10^{-9}$, but $\mathrm{CO}$ emission is weak or absent and would require a $\mathrm{CO}-\mathrm{H}_{2}$ conversion factor $\approx 10^{21} \mathrm{H}_{2} \mathrm{~cm}^{-2}\left(\mathrm{~K}-\mathrm{km} \mathrm{s}^{-1}\right)^{-1}$. This stands in marked contrast to the overluminous $\mathrm{CO}$ emission from the central molecular zone where the $N\left(\mathrm{H}_{2}\right) / W_{\mathrm{CO}}$ ratio is believed to be 3-10 times lower than the value near the Sun.

The physical properties and total mass of the molecular gas in the bulge remain to be determined by observation. We suggested that the atomic-molecular transition between the disk and bulge results from a weak UV radiation field inside the bulge with its older stellar population, encouraging $\mathrm{H}_{2}$ formation at relatively low number and column densities in otherwise atomic gas. Suppression of $\mathrm{CO}$ emission at brightness levels of $1-2 \mathrm{~K}$ (typical of existing survey detection limits) would require densities that are characteristic of mostly atomic $\mathrm{H}$ I clouds in the disk, $n(\mathrm{H}) \lesssim 30-50 \mathrm{~cm}^{-3}$. Information on the in situ number density in the absorbing molecular gas can be derived by observing $\mathrm{HCO}^{+}$, $\mathrm{HCN}$, or HNC in emission around the position of B1741-312, given the column densities measured in absorption and the fact that the emission brightness at the limit of detectability varies only as the $n(\mathrm{H})-N(\mathrm{X})$ product (Liszt \& Pety 2016).

ALMA Cycle 4 observations of a small handful of other suitably strong mm-wave continuum sources are underway, but none are as fortuitously positioned at such small longtitude and latitude as B1741-312. We note that even if the sort of detailed discussion presented here cannot currently be extended to other sightlines, existing 3D measurements of the reddening can be compared to $\mathrm{H} \mathrm{I}$ emission at bulge velocities to determine whether the atomic gas deficit seen here is a general phenomenon, as we would predict.

Acknowledgements. This paper makes use of the following ALMA data: ADS/JAO.ALMA\#2013.1.01194.S. ALMA is a partnership of ESO (representing its member states), NSF (USA) and NINS (Japan), together with NRC (Canada), NSC and ASIAA (Taiwan), and KASI (Republic of Korea), in cooperation with the Republic of Chile. The Joint ALMA Observatory is operated by ESO, AUI/NRAO and NAOJ. The National Radio Astronomy Observatory is a facility of the National Science Foundation operated under cooperative agreement by Associated Universities, Inc. This work was supported by the French program "Physique et Chimie du Milieu Interstellaire" (PCMI) funded by the Conseil National de la Recherche Scientifique (CNRS) and Centre National d'Etudes Spatiales (CNES). We thank Edwige Chapillon and Philippe Salomé for help with the ALMA data processing and the referee for helpful comments. HSL is grateful to the ENS and FREI for their hospitality during the completion of this manuscript.

\section{References}

Bialy, S., \& Sternberg, A. 2016, ApJ, 822, 83

Bitran, M., Alvarez, H., Bronfman, L., May, J., \& Thaddeus, P. 1997, ApJS, 125, 99

Bolatto, A. D., Wolfire, M., \& Leroy, A. K. 2013, Ann. Rev. Astrophys. Astron., 51, 207

Burton, W. B., \& Liszt, H. S. 1978, ApJ, 225, 815

Burton, W. B., \& Liszt, H. S. 1983, A\&AS, 52, 63

Churchwell, E., Babler, B. L., Meade, M. R., et al. 2009, PASP, 121, 213

Dame, T. M., \& Thaddeus, P. 2008, ApJ, 683, L143

Dickey, J. M., Kulkarni, S. R., Heiles, C. E., \& Van Gorkom, J. H. 1983, ApJS, 53,591

Fux, R. 1999, A\&A, 345, 787

Godard, B., Falgarone, E., Gerin, M., Hily-Blant, P., \& de Luca, M. 2010, A\&A, 520, A20

Heeschen, D. S. 1955, ApJ, 121, 569

Hensley, B. S., \& Draine, B. T. 2017, ApJ, 836, 179

HI4PI Collaboration, Ben Bekhti, N., Flöer, L., et al. 2016, A\&A, 594, A116

Koda, J., Scoville, N., \& Heyer, M. 2016, ApJ, 823, 76

Liszt, H. 2014a, ApJ, 783, 17

Liszt, H. 2014b, ApJ, 780, 10

Liszt, H. S. 2006, A\&A, 447, 533

Liszt, H. S. 2008, A\&A, 486, 467

Liszt, H. S., \& Burton, W. B. 1978, ApJ, 226, 790

Liszt, H., \& Lucas, R. 2001, A\&A, 370, 576

Liszt, H. S., \& Pety, J. 2016, ApJ, 823, 124

McClure-Griffiths, N. M., Dickey, J. M., Gaensler, B. M., et al. 2005, ApJS, 158, 178

Morris, M., \& Serabyn, E. 1996, ARA\&A, 34, 645

Robitaille, T. P., Churchwell, E., Benjamin, R. A., et al. 2012, A\&A, 545, A39

Rodriguez-Fernandez, N. J., Combes, F., Martin-Pintado, J., Wilson, T. L., \& Apponi, A. 2006, A\&A, 455, 963

Schlafly, E. F., \& Finkbeiner, D. P. 2011, ApJ, 737, 103

Schultheis, M., Chen, B. Q., Jiang, B. W., et al. 2014, A\&A, 566, A120

Sternberg, A., Le Petit, F., Roueff, E., \& Le Bourlot, J. 2014, ApJ, 790, 10 